\title{
Targeted delivery of reduced graphene oxide nanosheets using multifunctional ultrasound nanobubbles for visualization and enhanced photothermal therapy
}

This article was published in the following Dove Press journal:

International Journal of Nanomedicine

\author{
Zhao Liul,* \\ Jia Zhang ${ }^{2, *}$ \\ Yuhang Tian' \\ Lei Zhang' \\ Xue Han' \\ Qiucheng Wang' \\ Wen Cheng' \\ 'Department of Ultrasound, \\ Harbin Medical University Cancer \\ Hospital, Nangang District, Harbin \\ 150080, China; ${ }^{2}$ Key Laboratory of \\ Microsystems and Microstructure \\ Manufacturing, Ministry of Education, \\ Harbin Institute of Technology, Harbin \\ I50080, China \\ *These authors contributed equally \\ to this work
}

Correspondence: Wen Cheng Department of Ultrasound, Harbin Medical University Cancer Hospital, I 50 Haping Road, Nangang District, Harbin 150080, China

Tel/fax +86 45। 857। 8396

Email chengwen69@yahoo.com
Abstract: Ultrasound molecular imaging as a promising strategy, which involved the use of molecularly targeted contrast agents, combined the advantages of contrast-enhanced ultrasound with the photothermal effect of reduced graphene oxide (rGO).

Methods and results: The heparin sulfate proteoglycan glypican-3 (GPC3) is a potential molecular target for hepatocellular carcinoma (HCC). In this study, we covalently linked biotinylated GPC3 antibody to PEGylated nano-rGO to obtain GPC3-modified rGO-PEG (rGO-GPC3), and then combined rGO-GPC3 with avidinylated nanobubbles (NBs) using biotin-avidin system to prepare NBs-GPC3-rGO with photothermal effect and dispersibility, solubility in physiological environment. The average size of NBs-GPC3-rGO complex was $700.4 \pm 52.9 \mathrm{~nm}$ due to the polymerization of biotin-avidin system. Scanning electron microscope (SEM) showed NBs-GPC3-rGO attached to human hepatocellular carcinoma HepG2 cell. The ultrasound-targeted nanobubble destruction (UTND) technology make use of the physical energy of ultrasound exposure for the improvement of rGO delivery. Compared with other control groups, the highest nanobubble destruction efficiency of NBs-GPC3-rGO was attributed to the dissection effect of rGO on UTND. This is a positive feedback effect that leads to an increase in the concentration of rGO around the HepG2 cell. So NBs-GPC3-rGO using UTND and near-infrared (NIR) irradiation resulted in cell viability within $24 \mathrm{~h}, 48 \mathrm{~h}, 72 \mathrm{~h}$ lower than other treatment groups.

Conclusion: This work established NBs-GPC3-rGO as an ultrasonic photothermal agent due to its suitable size, imaging capability, photothermal efficiency for visual photothermal therapy in vitro.

Keywords: reduced graphene oxide, ultrasound-targeted nanobubble destruction, glypican-3, HepG2 cell, photothermal therapy

\section{Introduction}

Hepatocellular carcinoma (HCC) is the sixth most common malignant neoplasm in the world due to its high rates of recurrence and metastasis. ${ }^{1}$ A lot of progress has been made both in surgical and non-surgical therapies; however, patients are still subject to a very poor prognosis. ${ }^{1,2}$ Because of the propensity for metastasis, the 5-year survival rate of HCC patients is still very poor, and about $600,000 \mathrm{HCC}$ patients die each year. ${ }^{3}$ Therefore, a novel therapeutic method is of high importance. Photothermal therapy (PTT), a non-invasive, highly selective and potentially efficient cancer therapy, has attracted significant attention in recent years. ${ }^{4,5}$ In typical PTT, photosensitizing materials are utilized by cells to generate a localized temperature increase upon light absorption, leading to photoablation of the cancer cells and subsequent cell death. 
For this reason, the photosensitizing materials must possess a high selectivity to tumor cells to avoid killing the healthy cells. ${ }^{6}$ In addition, the material should be able to strongly absorb near-infrared (NIR) light, ${ }^{7}$ which is considered to be the optimal optical window for PTT due to a high transparency of biological tissue, blood and water. ${ }^{8,9}$

Recently, graphene has attracted extensive attention in many fields owing to its excellent properties. ${ }^{10-17}$ In biomedicines, the high specific surface area and multi-functionalization allow graphene to be widely used as a carrier for both drug and gene delivery. ${ }^{18-21}$ In addition, the high photothermal responsivity and low toxicity of reduced nanosized graphene oxide (nano-rGO) make it a promising photo-absorbing agent for PTT. ${ }^{6}$ Nevertheless, the current performance evaluation relies heavily on imaging techniques, such as CT, MRI and fluorescence imaging, which must be carried out several hours to days after PTT treatment. What is worse, previous results indicate that rGO does not have imaging capabilities during the tumor ablation process. As a result, there is still lack of photosensitizing materials, which possess the capabilities of both transporting and imaging in real time.

Contrast-enhanced ultrasound as a mature technology has been applied for HCC patients for years in many countries. Over recent decades, ultrasound molecular imaging based on the advantage of contrast-enhanced ultrasound has attracted significant attention in preclinical research of HCC diagnosis and therapy. ${ }^{22,23}$ It is a promising imaging strategy with great potential for improving diagnostic accuracy compared to conventional ultrasound imaging in $\mathrm{HCC}$ detection. ${ }^{24}$ With the rapid development of ultrasound molecular imaging bionanotechnology, the nanobubble (NB) as an ultrasound contrast agent has been established. Subsequently, NBs can access the extravascular space and provide unique advantages for targeted specific ultrasound imaging due to their small size, stability, and novel physical and surface properties. ${ }^{22}$ Recently, ultrasound-targeted NB destruction (UTND), as a safe and effective method, has been able to destruct contrast agents in ultrasound-irradiated locations, which plays a substantial role in improving the efficient delivery of exogenous genes and drugs noninvasively by sonoporation of NBs. ${ }^{22,23,25,26} \mathrm{As}$ a result, a novel contrast agent having a combination of $\mathrm{rGO}$ sheets with NBs can be synthesized, which has both ultrasound molecular imaging and photothermal capabilities; thus allowing monitoring of the PTT process in real time.

Herein, we synthesized a new theranostic nanoparticle containing nano-rGO-encapsulated NBs, which can achieve visualization and enhance the effect of PTT. In order to target the contrast agents, the composition was linked to a novel
HCC biomarker, the heparin sulfate proteoglycan glypican-3 (GPC3), which is highly expressed in most HCC patients' cancer cells, but not in normal adult tissues. ${ }^{27-33}$ Thus, given the HCC-specific nature of GPC3 expression, we hypothesized that ultrasound molecular imaging using GPC3-targeted NBs would allow transfecting of rGO sheets to the HCC cell membrane and highly accurate monitoring of the PTT process in real time. To the best of our knowledge, application of GPC3coated and rGO-loaded NBs as ultrasonic photothermal agent directed at HCC cells has not been reported elsewhere, and is considered innovative.

\section{Experimental section Materials and synthesis process} Synthesis of avidinylated NBs

The biotin-avidin system can be used as a link to integrate large amounts of biotin-labeled agents with avidinlyated NBs, due to its unique properties, which is extremely stable over a wide range of temperature and $\mathrm{pH}$ value. ${ }^{34-36}$ The avidinylated NBs were synthesized by a modified emulsification process. ${ }^{37,38}$ In brief, three types of liposomes, 1,2-distearoyl-sn-glycero-3-phosphocholine (DSPC); 1, 2-distearoyl-sn-glycero-3-phosphoethanolamine-N-[methoxy (polyethylene glycol)-2000] (DSPE-PEG2000); and 1,2distearoyl-sn-glycero-3-phosphoethanolamine-N-[biotinyl (polyethylene glycol)-2000] (DSPE-PEG2000-biotin), were purchased from Avanti Polar Lipids (Alabaster, AL, USA) and mixed in the molar ratio of 9:0.5:0.5. A certain amount of phospholipids were dissolved in the solvent of chloroform and methanol $(2: 1, \mathrm{v}: \mathrm{v})$, and then the mixture was transferred into a rotary vacuum evaporator to remove the solvent at $55^{\circ} \mathrm{C}$. Repeated dissolution and evaporation was carried out for three times. Then, the dried phospholipid compounds were hydrated in $5 \mathrm{~mL}$ of PBS ( $\mathrm{pH} 7.4$ ) at room temperature. The liposomal suspension was then transferred into a small sample vial, and the air above the solution was sucked and replaced with perfluoropropane $\left(\mathrm{C}_{3} \mathrm{~F}_{8}\right.$, Research Institute of Physical and Chemical Engineering of Nuclear Industry, Tianjin, China) gas. The admixture was then mechanically vibrated for 45 seconds in a dental amalgamator (YJT Medical Apparatuses and Instruments, Shanghai, China), and resuspended in $5 \mathrm{~mL}$ of sterile PBS. The supernatant was collected to acquire the non-targeted NBs. The NBs were subsequently washed with PBS and centrifuged at $800 \mathrm{rpm}$ for 3 minutes to remove the unbound lipids. Finally, the concentration of NBs was estimated to be $\sim 2 \times 10^{8} / \mathrm{mL}$ by using a hemocytometer (BürkerTürk, Wertheim, Germany). The size and dispersion of the NBs were detected by microscope (DM3000, Leica, Wetzlar, 
Germany) and the morphology of the NBs was examined using a transmission electron microscope (TEM) (JEM-1011, JEOL, Inc., Peabody, MA, USA).

To prepare fluorescence-labeled NBs, $50 \mu \mathrm{g}$ of FITClabeled avidin (SA, Beijing Biosynthesis Biotechnology Co., Ltd., Beijing, China) for each $1 \times 10^{8} / \mathrm{mL}$ NBs was added and incubated for 30 minutes at room temperature. ${ }^{39}$ The avidinylated NBs were washed with $3 \mathrm{~mL}$ PBS three times and then centrifuged at $800 \mathrm{rpm}$ for 3 minutes. FITC-labeled avidinylated NBs were observed by fluorescence microscope.

\section{Synthesis of PEG functionalized graphene oxide (rGO-PEG)}

Graphene oxide (GO) was synthesized using a modified Hummers' method. ${ }^{20,40-42}$ The nano-size GO (nano-GO) was obtained after immersion sonication for 2 hours. To functionalize the nano-GO, $1.2 \mathrm{~g}$ of sodium hydroxide $(\mathrm{NaOH})$ was added to the nano-GO suspension $(1 \mathrm{mg} / \mathrm{mL}, 10 \mathrm{~mL})$ and bath sonicated for 3 hours, followed by adjusting its $\mathrm{pH}$ value to one using hydrochloric acid (1 M). The mixture was neutralized and purified by repeated washing and centrifugation. Then, amine terminated six-arm branched PEG $(2 \mathrm{mg} / \mathrm{mL})$ was added into the abovementioned GO solution $(1 \mathrm{mg} / \mathrm{mL})$, and subjected to sonication for 5 minutes. N-(3-dimethylaminopropyl$\mathrm{N}^{\prime}$-ethylcarbodiimide) hydrochloride (EDC; Sigma-Aldrich Co., St Louis, MO, USA) was then added to the mixture in two equal portions to give a final concentration of $1 \mathrm{mg} / \mathrm{mL}$ in total, followed by sonication for another 1 hour. The solution was then centrifuged at $10,000 \mathrm{rpm}$ for 10 minutes in PBS for three times to remove any aggregates or multilayered GO sheets. The reaction mix was stirred overnight at room temperature, yielding a GO-PEG solution.

Hydrazine monohydrate was added into the nano-GO solution and heated at $80^{\circ} \mathrm{C}$ for 20 minutes to get aggregated nano-rGO sheets. These nano-rGO sheets were resuspended by bath sonication for 1 hour and then adjusted to a $\mathrm{pH}$ of 8.2 by adding $\mathrm{NaOH}$. Centrifugation and dialysis was necessary to remove any aggregated sheets and oxygenic functional groups. The supernatant was collected after centrifugation and washed three times with $100 \mathrm{kDa}$ molecular weight cutoff (MWCO) Millipore (EMD Millipore, Billerica, MA, USA) centrifuge filter at 4,000 rpm. Finally, nano-rGO-PEG solution was prepared and stored at $4^{\circ} \mathrm{C}$ for further usage. The covalent bonds in nano-GO-PEG and nano-rGO-PEG sheets were confirmed by various instrumental techniques such as Fourier transform infrared (FTIR, Nicolet 380, Thermo Electron Corporation, USA) analysis and X-ray diffraction (XRD) (Empyrean; Malvern Panalytical B.V., Almelo, the Netherlands) analysis.

\section{Synthesis of biotinylated rGO-GPC3}

Biotinylated cy7-conjugated GPC3 (Beijing Biosynthesis Biotechnology Co., Ltd.) antibody was dissolved in PBS as the stock solution ( $1 \mathrm{mg} / \mathrm{mL}$ ). A solution of $20 \mu \mathrm{L}$ of biotinylated cy7-conjugated anti-GPC3 was added to the rGO-PEG (20 $\mathrm{mg} / \mathrm{L}, 1 \mathrm{~mL}$ ) and the mixture was bath sonicated for 5 minutes. EDC was then added to the mixture to give a final concentration of $1 \mathrm{mg} / \mathrm{mL}$. The mixture was stirred overnight at room temperature. Excess biotinylated cy7-conjugated anti-GPC3 was removed by centrifuge filtration through Amicon centrifugal filters (EMD Millipore) with $100 \mathrm{kDa}$ MWCO and washed with distilled water for 4-5 times. UVvis spectra of nano-GO, nano-rGO, rGO-GPC3-cy7 and free cy7 were performed using a UV-vis spectrometer (Lambda 35, PerkinElmer, Waltham, MA, USA).

\section{Synthesis of NBs-GPC3-rGO}

Next, $20 \mu \mathrm{L}$ of biotinylated rGO-GPC3 per $10^{8} \mathrm{NBs}$ was added to the avidinylated NBs and incubated at $4{ }^{\circ} \mathrm{C}$ for 1 hour. The mixture was then washed with $\mathrm{PBS}$ to remove unbound rGO-GPC3 and obtain NBs-GPC3-rGO. The size distribution and the zeta potential of NBs, avidinlyated NBs, NBs-GPC3-rGO solution were determined by dynamic light scattering measurements using Zetasizer Nano ZS90 analyzer (Malvern Instruments Ltd., Malvern, UK).

\section{Detection of FITC-labeled biotinylated rGO-GPC3 conjugated with avidinylated NBs}

Since the emission of $\mathrm{Cy} 7$ is near infrared, we used another dye, FITC, to prove the linkage of NBs-GPC3-rGO by using luminescence image. For the linkage, FITC-labeled biotinylated anti-GPC3 antibody (Beijing Biosynthesis Biotechnology Co., Ltd.) was added in the GPC3-rGO solution and incubated in the dark area. The fluorescence intensity of the biotinylated FITC-labeled rGO-GPC3 bound to avidinylated NBs was assessed by a flow cytometer (Becton Dickinson Co., Ltd., Franklin Lakes, NJ, USA).

\section{Cell culture}

HepG2 cells, a human HCC cell line, were purchased from Shanghai Yu Bo Biotech Co. Ltd and were cultured in minimum Eagle medium (MEM, Invitrogen, Carlsbad, CA, USA) supplemented with 10\% FBS (Gibco, Carlsbad, CA, USA), and $1 \%$ penicillin/streptomycin. The cell cultures were maintained in a humidified atmosphere of $5 \% \mathrm{CO}_{2}$ at $37^{\circ} \mathrm{C}$ with the medium changed every other day. The total cell count was determined by a hemocytometer (Bürker-Türk). Exponentially growing cells were used for all experiments. 


\section{Targeted binding of NBs-GPC3-rGO to HepG2 cells} in vitro

The binding capacity of NBs-GPC3-rGO was tested on GPC3 receptor-positive HCC cells. HepG2 cells $\left(5 \times 10^{4} / \mathrm{mL}\right)$ were incubated in Costar six-well plates as monolayers in $90 \%$ MEM containing $10 \%$ FBS with $1 \%$ penicillin/streptomycin at $37^{\circ} \mathrm{C}$ in a humidified atmosphere containing $5 \% \mathrm{CO}_{2}$ for 24 hours. After that, NBs-GPC3-rGO $\left(1 \times 10^{8} / \mathrm{mL}\right)$ was added into the cell medium in the six-well plates and incubated with HepG2 cells. After 1 hour of static exposure, the medium was removed and cells were washed with PBS three times to remove the unbound NBs-GPC3-rGO. After fixation, dehydration, drying and other steps, the NBs-GPC3-rGO bound to the HepG2 cells were examined by scanning electron microscope (SEM) (S-4100L, Hitachi, Tokyo, Japan).

\section{Ultrasound destructibility of various nanoparticles in vitro}

Ultrasound-mediated NBs destructibility analysis was performed by a previously developed method. ${ }^{43}$ In brief, NBs were exposed to ultrasound at a frequency of $1 \mathrm{MHz}$ and an output intensity of $3.5 \mathrm{~W} / \mathrm{cm}^{2}$ using a $20-\mathrm{mm}$ probe (Institute of Ultrasound Imaging, Second Affiliated Hospital of Chongqing Medical University, Chongqing, China). There were three groups of NBs: blank (no sonication), avidinylated NBs and NBs-GPC3-rGO. Each ultrasound cycle included four frames of 30 seconds at a duty cycle of $50 \%$. After ultrasound exposure, the concentrations of various NBs were assessed on a hemocytometer (Bürker-Türk). Each type of sample was treated and measured three times.

\section{Cell toxicity assessment}

PEGylated nano-rGO toxicity was determined by Cell Counting Kit-8 (CCK-8) assay. HepG2 cells growing in log phase were seeded at a density of $8 \times 10^{3}$ cells/well $(100 \mu \mathrm{L}$ in volume per well) in 96-well assay plates for 24 hours and then treated with various concentrations of PEGylated nano-rGO for 48 hours. After washing the culture with PBS solution three times, $10 \mu \mathrm{L}$ of CCK-8 solution was added to each well for 2 hours. The cell toxicity was measured with a microplate reader (BioTek, Winooski, VT, USA) at an absorbance of $450 \mathrm{~nm}$.

\section{Detection of reactive oxygen species (ROS)}

Under NIR (808 nm) irradiation, extracellular ROS may be generated from two components, which are pure water and NBs-GPC3-rGO solution (20 mg/L). The extracellular ROS of a sample was detected using 1,3-diphenylisobenzofuran (DPBF) as a probe. In brief, $30 \mu \mathrm{L}$ of N, N-dimethylformamide solution containing DPBF $(1 \mathrm{mg} / \mathrm{mL})$ was added into a control group of $3 \mathrm{~mL}$ of the above two solutions. Then, these two solutions were irradiated under a NIR laser for various durations. After centrifugation, the optical absorbance of each solution at $410 \mathrm{~nm}$ was measured on a spectrophotometer (U-4100, Hitachi).

\section{In vitro PTT after UTND}

HepG2 cells were plated in 96-well plates at a density of $8 \times 10^{3}$ cells/well in MEM complete media. After 1 hour of incubation at $37^{\circ} \mathrm{C}$ with NBs-GPC3-rGO, or control, the vials containing cell pellets were stored in ice until irradiation. The cells were incubated with NBs-GPC3-rGO $(20 \mathrm{mg} / \mathrm{L})$ or control at $37^{\circ} \mathrm{C}$ in a humidified atmosphere containing $5 \% \mathrm{CO}_{2}$. After 1 hour of incubation, the wells were washed with PBS three times. The cell wells were divided into seven groups: 1) HepG2 cells with MEM only (negative control); 2) UTND only; 3) laser only; 4) NBs-GPC3-rGO only; 5) NBs-GPC3-rGO + UTND; 6) NBs-GPC3-rGO + laser; 7) NBs-GPC3-rGO + UTND + laser. UTND parameters were as follows: frequency $(1 \mathrm{MHz})$; output intensity $\left(1.0 \mathrm{~W} / \mathrm{cm}^{2}\right)$; and irradiation (1 minute/well). Laser parameters were as follows: wavelength $(808 \mathrm{~nm})$; output intensity $\left(2.0 \mathrm{~W} / \mathrm{cm}^{2}\right)$ and irradiation (10 minutes/well). After the process, $100 \mu \mathrm{L}$ of fresh medium was then added into each well. The plates were kept in the incubator for 24, 48 and 72 hours. Cell viability was estimated by the CCK-8 assay as described.

\section{Statistical analysis}

The SPSS 13.0 software package (SPSS Inc., Chicago, IL, USA) was used for statistical analysis. The level of significance in all statistical analyses was set at a probability of $P<0.05$. Data were presented as mean $\pm \mathrm{SD}$. The various groups were analyzed by analysis of variance and Student's $t$-tests.

\section{Results and discussion}

The noninvasive ultrasound molecular imaging combined with the advantages of contrast-enhanced ultrasound can characterize neoplastic processes on a molecular level. It's gaining acceptance is due in part to some of the inherent advantages of ultrasound, namely its lack of ionizing radiation, noninvasiveness, and high spatial and temporal sensitivity. It has many potential applications in the early detection of cancer, monitoring tumor characterization treatment response and guiding cancer therapies. Ultrasound molecular imaging contrast agents also have potential applications in the field of hyperthermia for the delivery of chemotherapeutic drugs and gene therapy. NBs as one of the promising contrast agents 
were widely used in drug delivery and they were also used to increase the location concentration of carrier loads upon ultrasonication. Herein, the avidinylated NBs were synthesized by a modified emulsification process (Figure 1). ${ }^{37,38}$ For the synthesis, DSPE-PEG2000 and DSPE-PEG2000biotin were chosen as self-assembled shell of the NBs to encapsulate $\mathrm{C}_{3} \mathrm{~F}_{8}$ gas. Also, they played a key role in stable dispersion of loads, which will be explained in detail later in the paper. These liposome NBs in suspension are a milk white color with a diameter of $292.2 \pm 13.3 \mathrm{~nm}$ (Figure 2A and Figure S1A and D) with a concentration of $\sim 2 \times 10^{8} / \mathrm{mL}$. TEM image (Figure 2A inset) shows the spherical morphology of the NBs and confirms the range of size distribution. To prove the avidinylation of NBs, green dye FITC-labeled avidinylated NBs were synthesized and characterized by fluorescence microscopy. Figure $2 \mathrm{C}$ shows the green fluorescence emission from different parts, which correlates with the NB locations (Figure 2B), indicating the successful avidinylation of NBs.
Meanwhile, the size distribution of these avidinylated of NBs increased to $467.6 \pm 11.5 \mathrm{~nm}$ (Figure S1B and E).

GO was prepared by a modified Hummers' method ${ }^{42}$ with an initial lateral size of $\sim 5 \mu \mathrm{m}$ and single layer. To make it fit for injection, nano-GO sheets were obtained by horn ultrasonication. Figure 3A shows a typical AFM image of nano-GO sheets with a size of $50 \pm 13 \mathrm{~nm}$. Then, amine-terminated sixarm branched PEG was covalently linked to the carboxylic acid group by amide reaction, which makes the nano-GO-PEG soluble and stable dispersive in buffer solution. Furthermore, hydrazine monohydrate was added into the nano-GO-PEG solution and heated at $80^{\circ} \mathrm{C}$ for 20 minutes. The color of this solution gradually changed from yellow to black during the heating, indicating the reduction of GO and formation of nano-rGO-PEG. UV-vis spectra of both nano-GO-PEG and nano-rGO-PEG are shown in Figure 3B; the shift of absorption peak (from $\sim 230$ to $\sim 275 \mathrm{~nm}$ ) and an increased absorption in visible and NIR region (Figure 3B inset) also confirm the

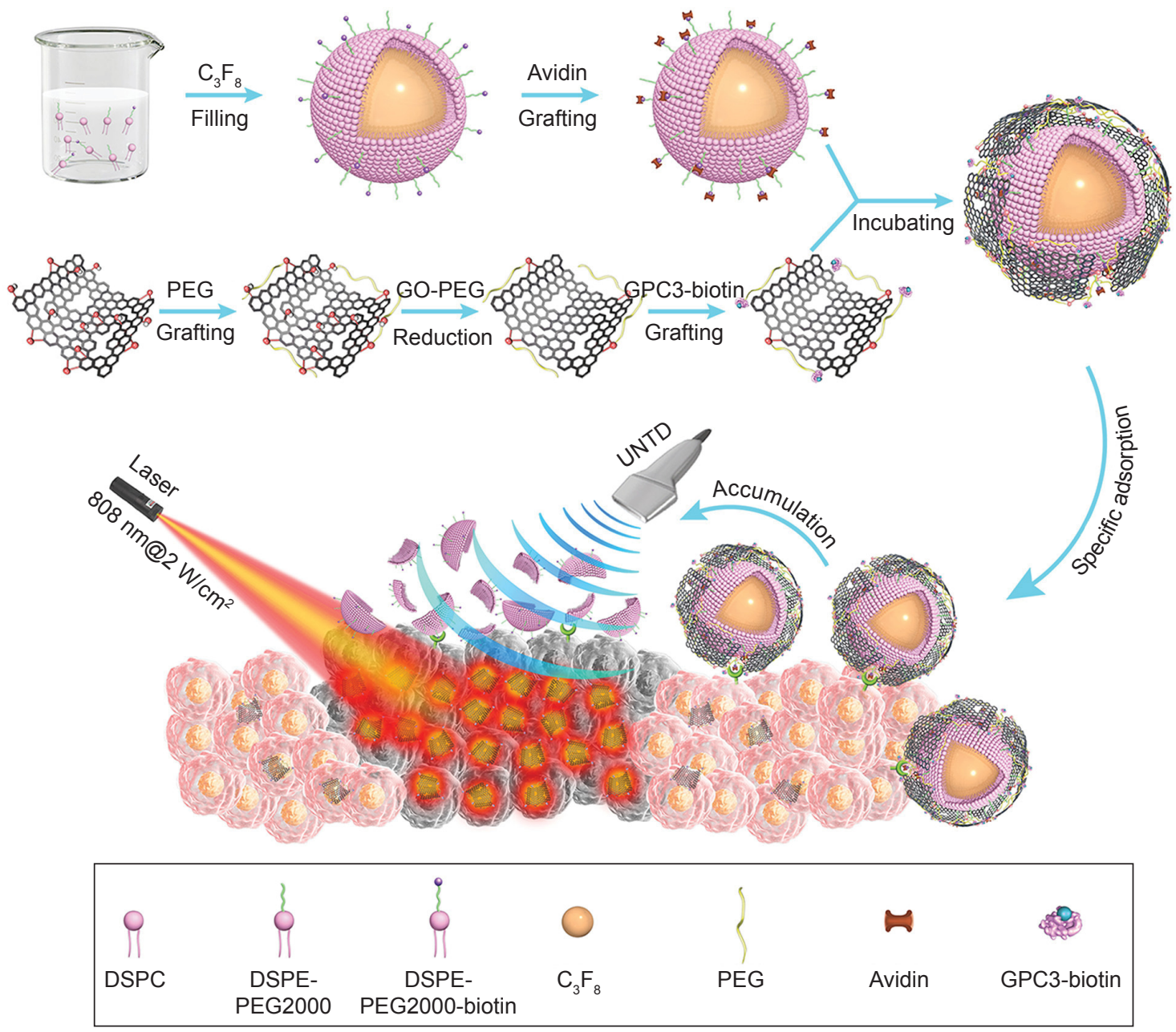

Figure I Schematic diagram of synthesis of NBs-GPC3-rGO constructed for rGO targeted delivery and photothermal therapy.

Abbreviations: DSPC, I, 2-distearoyl-sn-glycero-3-phosphocholine, DSPE-PEG2000, I, 2-distearoyl-sn-glycero-3-phosphoethanolamine-N-[methoxy(polyethylene glycol)2000]; DSPE-PEG2000-biotin, I, 2-distearoyl-sn-glycero-3-phosphoethanolamine-N-[biotinyl (polyethylene glycol)-2000)]; GPC3, heparin sulfate proteoglycan glypican-3; UTND, ultrasound-targeted nanobubbles destruction. 


\section{A}

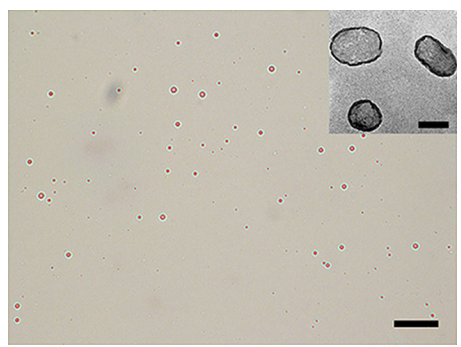

D

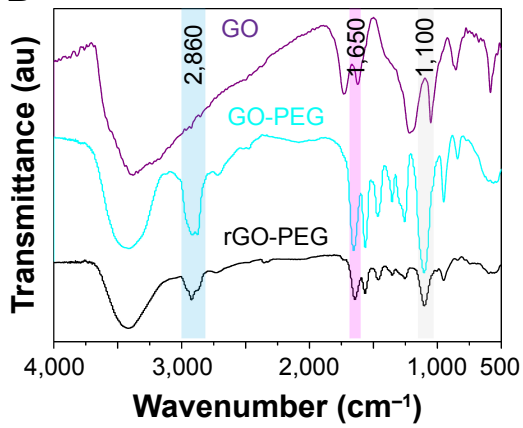

G

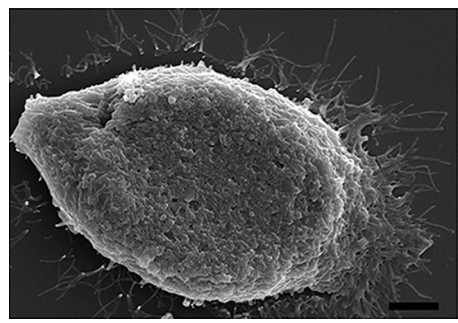

B

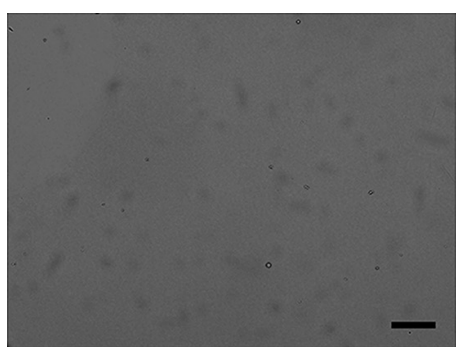

E

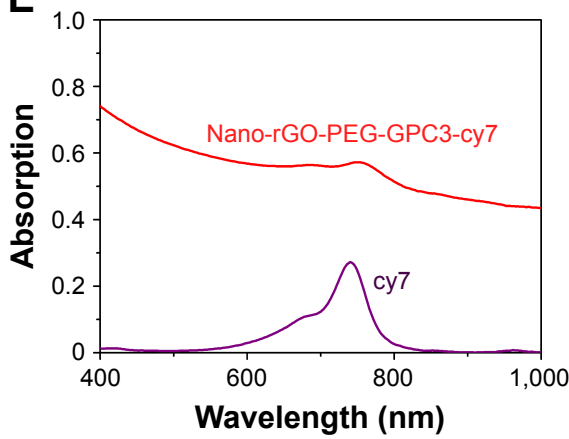

H

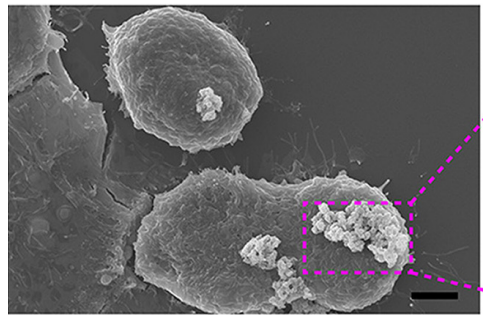

C

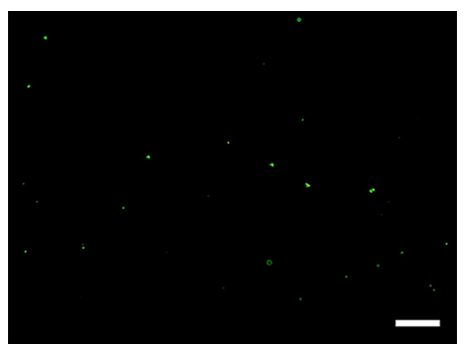

$\mathbf{F}$
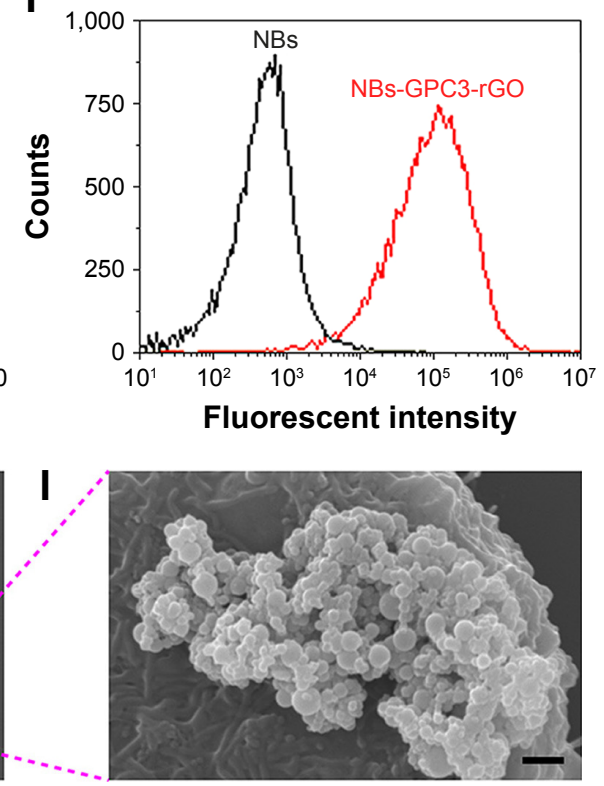

Figure 2 Characterization of nanobubbles, proof the chains are convelent together.

Notes: Bar in $\mathbf{A}-\mathbf{C}$ is $10 \mu \mathrm{m}$, inset in Figure $2 \mathrm{~A}$, bar $=100 \mathrm{~nm}$, bar in $\mathbf{G}$ and $\mathbf{H}=4 \mu \mathrm{m}$, bar in $\mathbf{I}=0.6 \mu \mathrm{m}$. (A) Nanobubbles (NBs) in suspension were milk white in color. Pure NB solution was observed under a light microscope (magnification, I,000x). (B) Avidinylated nanobubbles were observed under a light microscope (magnification, I,000x). (C) Nanobubbles conjugated with FITC-labeled avidin under fluorescence microscopy (magnification, I,000x). The surface of nanobubbles appeared green under fluorescence microscopy, indicating that FITC-labeled avidin was packaged on the NB surface. (D) Fourier transform infrared (FTIR) spectra of graphene oxide-polyethylene glycol (GO-PEG) and reduced graphene oxide (rGO)-PEG. The GO-PEG and rGO-PEG sample was filtered over three times through a I00 kDa molecular weight cutoff (MWCO) filter to completely remove any unconjugated PEG (I0 kDa). (E) A UV-vis-near-infrared (NIR) spectrum of nano-rGO-PEG-GPC3-cy7 and free cy7 dye solution. (F) Binding efficiency of biotinylated FITC-labeled rGO-PEG-GPC3 with avidinylated NBs was determined by flow cytometry. Comparison of the fluorescence intensities for NBs (black line, control) and FITC-labeled NBs-GPC3-rGO (red line) implies successful binding of rGO-PEG-GPC3 with avidinylated NBs. (G) Scanning electron microscopy revealed normal surface morphology of the Hep G2 cells (4,000x). Under scanning electron microscopy, targeted NBs-GPC3-rGO were clustered on the surface of the Hep G2 cells ( $\mathbf{H}$ and $\mathbf{I}, 4,000 \times$ and $10,000 \times$, respectively).

Abbreviation: GPC3, heparin sulfate proteoglycan glypican-3.
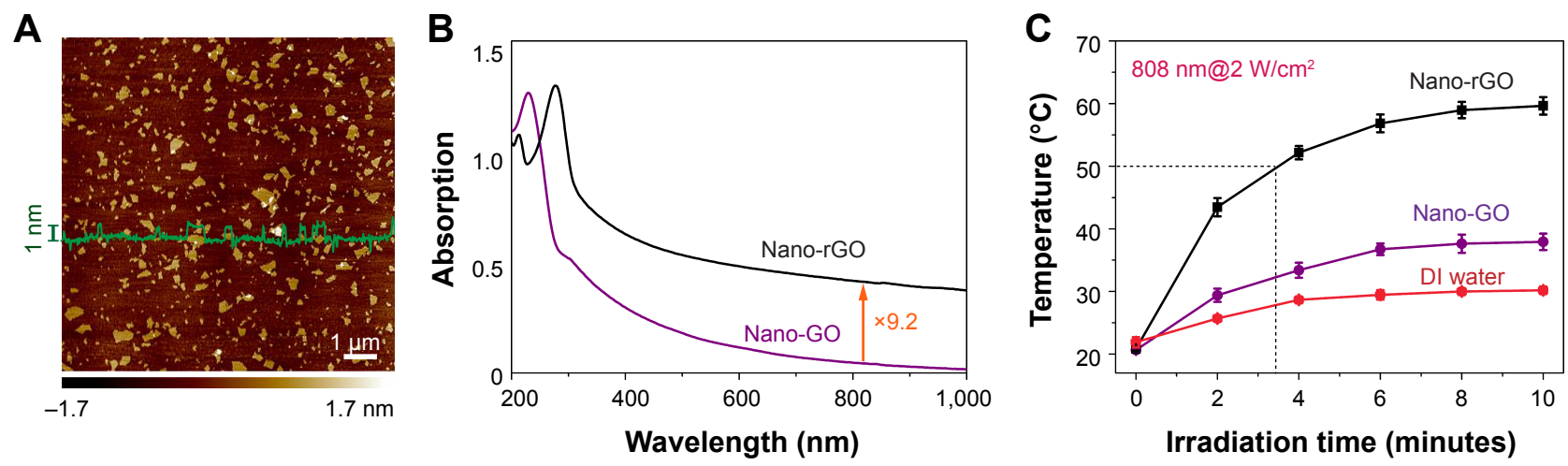

Figure 3 Characterization of reduced graphene oxide ( $\mathrm{rGO}$ ) sheets. (A) An AFM image of reduced nanographene oxide (nano-rGO). The image is on a I0 nm height scale. (B) A UV-vis-near-infrared (NIR) spectrum of nano-GO-polyethylene glycol (PEG) and nano-rGO-PEG solution. (C) Photothermal heating curves of the nano-rGO-PEG and nano-GO-PEG solutions and the deionized water (DIW) exposed to the $808 \mathrm{~nm}$ laser at a power density of $2 \mathrm{~W} / \mathrm{cm}^{2}$. Black curve is I mL of solutions with $20 \mathrm{mg} / \mathrm{L}$ concentration of nano-rGO-PEG, red curve is nano-GO-PEG and dark blue curve is DIW. 
recovery of conjugated $\pi$ structure of graphene. ${ }^{44,45}$ Raman spectroscopy of nano-GO and nano-rGO was performed at room temperature using a Raman Microprobe (LabRAM HR800; Horiba Scientific, Jobin-Yvon, France) (Figure S2). For the nano-rGO-PEG sample, there was one peak at $\sim 21.69^{\circ}$ in XRD pattern, indicating that most oxygen functional groups were removed thereby leading to a successful reduction of GO (Figure S3). ${ }^{46,47}$ As absorption of NIR increased, about 9.2 times at $808 \mathrm{~nm}$ (Figure 3B), the photothermal heating became more effective, which is one of the key elements to phototherapy. The new broad peak that appeared at $\sim 2,860 \mathrm{~cm}^{-1}$ both in nano-GO-PEG and nano-rGO-PEG samples in the FTIR spectra (Figure 2D) can be assigned to the symmetric stretching mode of the methylene groups of the PEG, indicating the PEG molecules were successfully conjugated to $\mathrm{GO}$ sheets, and such conjugation is quite stable even after chemical reduction. In addition, a strong peak at $\sim 1,100 \mathrm{~cm}^{-1}$ in the nano-GO-PEG sample may be attributed to the vibration of $-\mathrm{C}-\mathrm{O}-$ groups of PEG. The strong peaks at $\sim 3,400 \mathrm{~cm}^{-1}$ and $1,650 \mathrm{~cm}^{-1}$ were probably due to many more $-\mathrm{OH}$ groups in nano-GO-PEG compared that in nano-rGO-PEG sample.

After conjugation to PEG, the nano-rGO-PEG solution was grafted with cy7-labled GPC3 biotin solution. The UVvis spectra of pure cy 7 dye showed a strong absorption peak at $749 \mathrm{~nm}$ with a small shoulder (Figure 2E), which was also visible after grafting with nano-rGO-PEG, confirming the conjugation of GPC3-Cy7 to the PEG. Furthermore, the fluorescence intensities of avidinylated NBs after incubation with FITC-labeled biotinylated rGO-GPC3 indicated that about $86.5 \%$ of avidinylated NBs were successfully conjugated with biotinylated rGO-GPC3 (Figure 2F). Finally, the size distribution increased to $700.4 \pm 52.9 \mathrm{~nm}$ (Figure S1C and F). The gradually increasing size of NBs also gives evidence for successful connecting of different parts. In addition, the zeta potential of NBs-GPC3-rGO $(-18.74 \pm 2.02 \mathrm{mV})$ was lower than that of the NBs $(-24.39 \pm 2.40 \mathrm{mV})$ indicating a slight decrease in its stability and dispersivity, as shown in Figure S4.

To evaluate the photothermal heating performance, the rise in temperature was plotted as the function of irradiation time of deionized water (DI water), nano-GO (20 mg/L) and nano-rGO $(20 \mathrm{mg} / \mathrm{L})$ at a power density of $2 \mathrm{~W} / \mathrm{cm}^{2}$ of NIR $(808 \mathrm{~nm})$ shown in Figure 3C. The temperature of DI water increased slowly from $23^{\circ} \mathrm{C}$ to $30^{\circ} \mathrm{C}$ within 10 minutes, while that of the nano-GO solution exhibited a similar behavior with maximum value of $39^{\circ} \mathrm{C}$ within 10 minutes. This can be explained by the very weak absorption of DI water and
nano-GO solution at a wavelength of $808 \mathrm{~nm}$ (Figure 3B). By contrast, the temperature of nano-rGO solution increased rapidly to $60^{\circ} \mathrm{C}$ at the same condition. Significantly, it readily reached $50^{\circ} \mathrm{C}$ (threshold of photoablation) within 3.4 minutes compared with that of 5 minutes in Dai's work, ${ }^{6}$ indicating a more efficient photothermal heating of our nano-rGO solution. Furthermore, the nano-rGO solution showed a monotonical enhancement of photothermal heating performance with increasing concentration. The time required to reach the temperature of $50^{\circ} \mathrm{C}$ was about $4.7,3.4$ and 2.6 minutes for the concentrations of 10,20 and $40 \mathrm{mg} / \mathrm{L}$, respectively. The more nano-rGO the sheets contains, the more absorption of NIR, which is transformed to heat.

Furthermore, the avidinated NBs were linked to the nano-rGO-PEG-GPC3-biotin by the avidin-biotin system and formed the expected contrast-targeted delivery particles. (Figure 1). Then, it was incubated with the HepG2 cells in the Costar six-well plates. SEM image shows that a large amount of NBs-GPC3-rGO was targeted to the surface of HepG2 cells successfully (Figures 1, 2H and I bottom panel), while the original cell had a very clean surface (Figure $2 \mathrm{G}$ ).

Ultrasound-targeted NB destructibility was assessed for avidinylated NBs, NBs-GPC3-rGO and Blank (NB solution without sonication). Figure $4 \mathrm{~A}$ shows the concentration of NBs as a function of sonication time, from which the values of attenuation half-life ( $\mathrm{t} / 2)$ were 85 seconds and 54 seconds for the avidinylated NBs and NBs-GPC3-rGO, respectively, with significant difference between the two types of NBs and the Blank group $(P<0.05)$. However, there was a statistically significant difference between avidinylated NBs and NBsGPC3-rGO $(P<0.05)$. The highest NB destruction efficiency of NBs-GPC3-rGO may be attributed to the dissection effect of $\mathrm{rGO}$ on UTND.

Further, we examined the biocompatibility of covalently PEGylated nano-rGO with HepG2 cell lines. The half maximal inhibitory concentration of nano-rGO-PEG was found to be $\sim 61 \mathrm{mg} / \mathrm{L}$ for human HCC cell line HepG2 (Figure 4B). It is smaller than previously reported, however PEGylated nanorGO decreased the viability of MCF-7 cells (a human breast cancer cell line) at $\sim 80 \mathrm{mg} / \mathrm{L}$. It shows that nano-rGO-PEG has a satisfactory toxicity assessment result. So, the concentration of nano-rGO should be much lower than $61 \mathrm{mg} / \mathrm{L}$ to ensure that the cell-killing ability comes from PTT and not from the toxicity itself. Also, the concentration-depended photothermal efficiency should be considered (Figure S5). As a result, a moderate concentration of $20 \mathrm{mg} / \mathrm{L}$ was taken except for special instruction while ensuring the better photothermal 

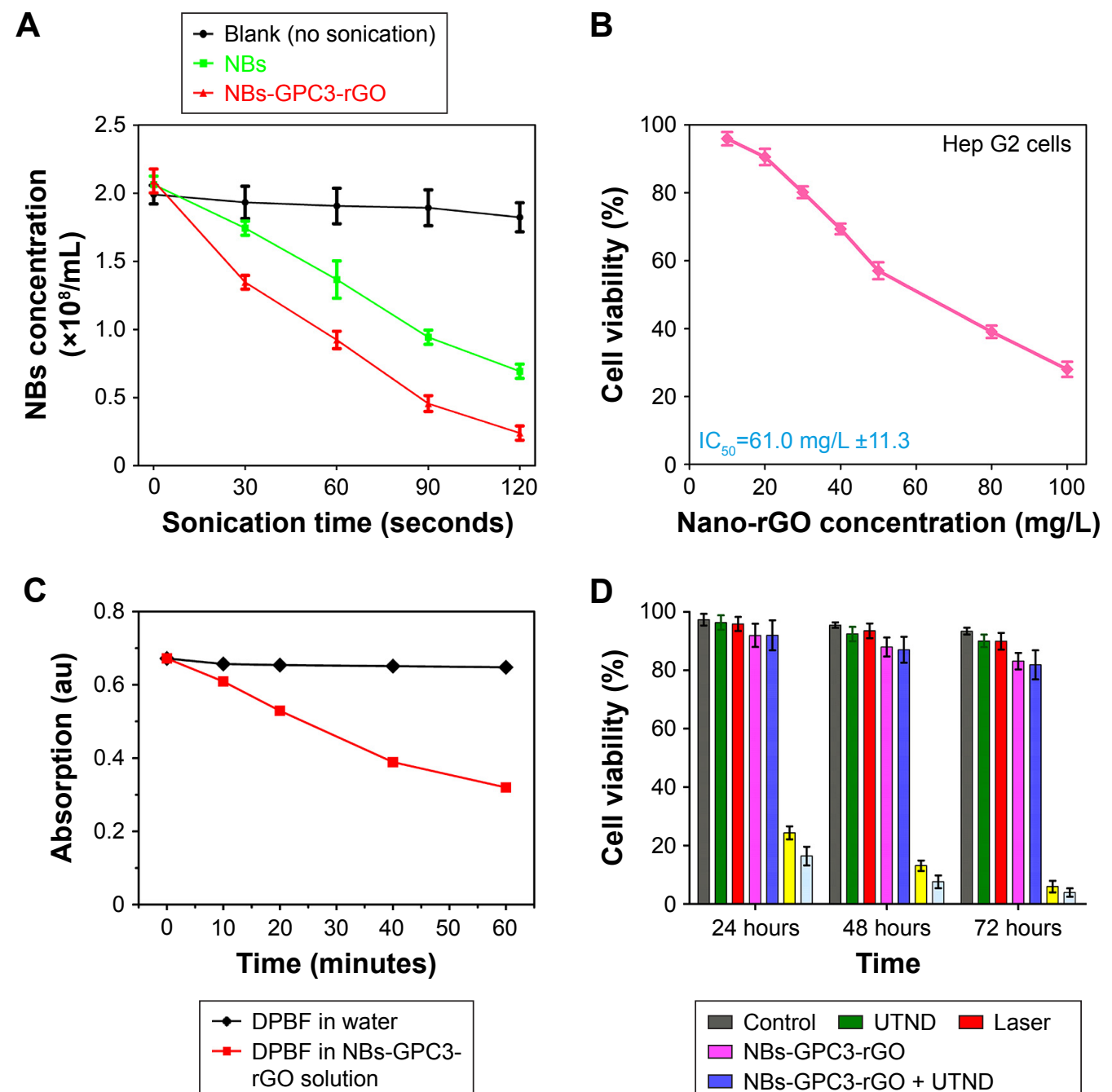

$\square$ Control $\square$ UTND $\square$ Laser
$\square$ NBs-GPC3-rGO
$\square$ NBs-GPC3-rGO + UTND
$\square$ NBs-GPC3-rGO + laser
$\square$ NBs-GPC3-rGO + UTND + laser

Figure 4 Quantitative growth inhibition assay in HepG2 cells after different treatments.

Notes: (A) Ultrasound-targeted destruction of nanobubbles (NBs)-GPC3-reduced graphene oxide (rGO), compared with Blank group, and avidinylated NBs. (B) Cellular viability data based on Cell Counting Kit-8 (CCK-8) for Hep G2 cells incubated for 48 hours with PEGylated nano-rGO. (C) Absorption spectra of the I, 3-diphenylisobenzofuran (DPBF) probe under different irradiation times. (D) Cellular viability effect of Hep $\mathrm{G} 2$ cells with different treatments. The viability rate of cells was determined by CCK-8 assay at 24, 48 and 72 hours after different treatments. Data are represented as mean \pm SD ( $n=7$ ). The cell viability rate of the NBs-GPC3-rGO + laser (group 6) and the NBs-GPC3-rGO + ultrasound-targeted NB destruction (UTND) + laser (group 7) are significantly lower than those of the other groups (P<0.05).

Abbreviations: DPBF, I, 3-diphenylisobenzofuran; GPC3, heparin sulfate proteoglycan glypican-3.

efficiency (Figure S5) and the lower cytotoxicity (Figure 4B) of nano-rGO in our experiments.

Moreover, photodynamic therapy (PDT) is a type of therapeutic approach that requires a photosensitizer to generate ROS to eliminate cancer tissues. ${ }^{48,49}$ For this purpose, the extracellular ROS generation of a sample was detected using DPBF as a probe. A decrement of DPBF characteristic absorption could be observed, accordingly. As shown in Figure 4C, the absorbance of the DPBF solution with NBs-GPC3-rGO decreased significantly as irradiation time went on, suggesting the sufficient ROS level was induced by NBs-GPC3-rGO. By contrast, the pure water led to very limited ROS generation with the same laser irradiation. Therefore, NBs-GPC3-rGO was used as a photosensitizer for executing PDT, which can be used as a supplementary treatment for PTT.

Finally, the effect of thermal therapy of our samples was evaluated by index of cell viability using CCK-8 assay. Figure 4D shows the cell viability rates of different treatment groups at 24, 48 and 72 hours. Almost all HepG2 cells incubated with NBs-GPC3-rGO were destroyed due to irradiation with $2 \mathrm{~W} / \mathrm{cm}^{2}$ NIR light for 10 minutes with (group 7) or without (group 6) UTND, while all other groups showed near $100 \%$ viability. Compared with other treatment groups, the cell viability rates of groups (6) and (7) showed 
significantly lower efficiency $(P<0.05)$, suggesting that NBs-GPC3-rGO with laser irradiation was the main factor of killing cells. Although there was no statistical difference between groups 6 and $7(P>0.05)$, UTND had a positive effect on laser irradiation in reducing cell viability.

Upon the UTND, the NBs were found to be destroyed within a short time (Figure 4A). The rGO nanosheets were known as nano-knife or nano-blade and were used to cut bacteria and cell membrane with their sharp edges..$^{50}$ Analogously, rGO nanosheet would also perform as a blade to cut the NBs, especially upon ultrasonication. As a result, the UTND efficiency is significantly improved by $50 \%$ at the same sonication time (Figure 4A) after linking the rGO nanosheets. Moreover, a large number of rGO sheets were covered with NBs through avidin-biotin system and kept in close contact with each other. When NBs-GPC3-rGO are destroyed using UTND, rGO-GPC3 can be released to increase the concentration of $\mathrm{rGO}$ around the HepG2 cells. rGO-GPC3 can be attached to the surface of the HepG2 cells due to the targeting effect of GPC 3 antibodies. The nano-rGO solutions exhibited a concentration-dependent photothermal heating effect, ${ }^{6}$ which is in accordance with our results (Figures $3 \mathrm{C}$ and S5). In addition, UTND facilitates the transfer of extracellular molecules into cells via sonoporation. Therefore, in terms of the cell viability test, we noticed that the PTT effect of NBs-GPC3-rGO is the best compared with other groups, and its 72-hour cell viability rate is $2.26 \%$ (Figure $4 \mathrm{D}$ ).

\section{Conclusion}

In summary, we have developed a new ultrasound contrast agent containing nano-rGO encapsulated NBs, which can achieve visualization and enhanced PTT. GPC3 works as a target probe molecule to specific sites on the surface of HepG2 cells. NBs-GPC3-rGO was destroyed under the ultrasound, which boosted the local concentration of rGO thereby enhancing the photothermal ablation of cancer cells under the NIR irradiation. As a result, within 72 hours, viability rate of cancer cells treated by PTT was approximately $2.26 \%$ under UTND and NIR laser treatment. Our results lay the foundation for further study and clinical application of GPC3-targeted ultrasound imaging in noninvasive surveillance during the process of PTT using rGO.

\section{Acknowledgments}

This work was supported by the National Natural Science Foundation of China (grant nos 81571682 and 61771156),
HAI YAN Science Foundation of Harbin Medical University Cancer Hospital (grant no JJQN2018-18), Open Found of Key Laboratory of Microsystems and Microstructure Manufacturing Ministry of Education (HIT) (grant no 2016KM010).

\section{Disclosure}

The authors report no conflicts of interest in this work.

\section{References}

1. Global Burden of Disease Cancer Collaboration; Fitzmaurice C, Allen C, Barber RM, et al. Global, Regional, and National Cancer Incidence, Mortality, Years of Life Lost, Years Lived With Disability, and Disability-Adjusted Life-years for 32 Cancer Groups, 1990 to 2015 : A Systematic Analysis for the Global Burden of Disease Study. JAMA Oncol. 2017;3(4):524-548.

2. Song P, Tobe RG, Inagaki Y, et al. The management of hepatocellular carcinoma around the world: a comparison of guidelines from 2001 to 2011. Liver Int. 2012;32(7):1053-1063.

3. Yu J, Xu QG, Wang ZG, et al. Circular RNA cSMARCA5 inhibits growth and metastasis in hepatocellular carcinoma. J Hepatol. 2018; 68(6):1214-1227.

4. Chen Z, Wang Q, Wang H, et al. Ultrathin PEGylated W18O49 nanowires as a new $980 \mathrm{~nm}$-laser-driven photothermal agent for efficient ablation of cancer cells in vivo. Adv Mater. 2013;25(14):2095-2100.

5. Yang K, Yang G, Chen L, et al. FeS nanoplates as a multifunctional nano-theranostic for magnetic resonance imaging guided photothermal therapy. Biomaterials. 2015;38:1-9.

6. Robinson JT, Tabakman SM, Liang Y, et al. Ultrasmall reduced graphene oxide with high near-infrared absorbance for photothermal therapy. J Am Chem Soc. 2011;133(17):6825-6831.

7. Liu Y, Ashton JR, Moding EJ, et al. A Plasmonic Gold Nanostar Theranostic Probe for In Vivo Tumor Imaging and Photothermal Therapy. Theranostics. 2015;5(9):946-960.

8. Zha Z, Yue X, Ren Q, Dai Z. Uniform polypyrrole nanoparticles with high photothermal conversion efficiency for photothermal ablation of cancer cells. Adv Mater. 2013;25(5):777-782.

9. Markovic ZM, Harhaji-Trajkovic LM, Todorovic-Markovic BM, et al. In vitro comparison of the photothermal anticancer activity of graphene nanoparticles and carbon nanotubes. Biomaterials. 2011;32(4): 1121-1129.

10. Bitounis D, Ali-Boucetta H, Hong BH, Min DH, Kostarelos K. Prospects and challenges of graphene in biomedical applications. Adv Mater. 2013;25(16):2258-2268

11. Feng L, Liu Z. Graphene in biomedicine: opportunities and challenges. Nanomedicine. 2011;6(2):317-324.

12. Feng L, Zhang S, Liu Z. Graphene based gene transfection. Nanoscale 2011;3(3):1252-1257.

13. Yang X, Wang Y, Huang X, et al. Multi-functionalized graphene oxide based anticancer drug-carrier with dual-targeting function and $\mathrm{pH}$ sensitivity. J Mater Chem. 2011;21(10):3448-3454.

14. Ma X, Tao H, Yang K, et al. A functionalized graphene oxide-iron oxide nanocomposite for magnetically targeted drug delivery, photothermal therapy, and magnetic resonance imaging. Nano Res. 2012;5(3):199-212.

15. Yang K, Feng L, Shi X, Liu Z. Nano-graphene in biomedicine: theranostic applications. Chem Soc Rev. 2013;42(2):530-547.

16. Shen H, Zhang L, Liu M, Zhang Z. Biomedical applications of graphene. Theranostics. 2012;2(3):283-294

17. Shi X, Gong H, Li Y, Wang C, Cheng L, Liu Z. Graphene-based magnetic plasmonic nanocomposite for dual bioimaging and photothermal therapy. Biomaterials. 2013;34(20):4786-4793.

18. Sun X, Liu Z, Welsher K, et al. Nano-Graphene Oxide for Cellular Imaging and Drug Delivery. Nano Res. 2008;1(3):203-212. 
19. Zhang L, Xia J, Zhao Q, Liu L, Zhang Z. Functional graphene oxide as a nanocarrier for controlled loading and targeted delivery of mixed anticancer drugs. Small. 2010;6(4):537-544.

20. Liu Z, Robinson JT, Sun X, Dai H. PEGylated nanographene oxide for delivery of water-insoluble cancer drugs. J Am Chem Soc. 2008;130(33): 10876-10877.

21. Wang Y, Wang K, Zhao J, et al. Multifunctional mesoporous silicacoated graphene nanosheet used for chemo-photothermal synergistic targeted therapy of glioma. J Am Chem Soc. 2013;135(12):4799-4804.

22. Kaneko OF, Willmann JK. Ultrasound for molecular imaging and therapy in cancer. Quant Imaging Med Surg. 2012;2(2):87.

23. Mullick Chowdhury S, Wang TY, Bachawal S, et al. Ultrasound-guided therapeutic modulation of hepatocellular carcinoma using complementary microRNAs. J Control Release. 2016;238:272-280.

24. Zheng SG, Xu HX, Liu LN. Management of hepatocellular carcinoma: The role of contrast-enhanced ultrasound. World J Radiol. 2014;6(1): 7-14.

25. Wu M, Zhao H, Guo L, et al. Ultrasound-mediated nanobubble destruction (UMND) facilitates the delivery of A10-3.2 aptamer targeted and siRNA-loaded cationic nanobubbles for therapy of prostate cancer. Drug Deliv. 2018;25(1):226-240.

26. Song Z, Wang Z, Shen J, Xu S, Hu Z. Nerve growth factor delivery by ultrasound-mediated nanobubble destruction as a treatment for acute spinal cord injury in rats. Int J Nanomedicine. 2017;12:1717-1729.

27. Yang X, Liu H, Sun CK, et al. Imaging of hepatocellular carcinoma patient-derived xenografts using ${ }^{89} \mathrm{Zr}$-labeled anti-glypican-3 monoclonal antibody. Biomaterials. 2014;35(25):6964-6971.

28. Hsu HC, Cheng W, Lai PL. Cloning and expression of a developmentally regulated transcript MXR7 in hepatocellular carcinoma: biological significance and temporospatial distribution. Cancer Res. 1997;57(22): 5179-5184.

29. Gao W, Kim H, Feng M, et al. Inactivation of Wnt signaling by a human antibody that recognizes the heparan sulfate chains of glypican-3 for liver cancer therapy. Hepatology. 2014;60(2):576-587.

30. Filmus J. Glypicans in growth control and cancer. Glycobiology. 2001; 11(3):19R-23R.

31. Shirakawa H, Kuronuma T, Nishimura Y, et al. Glypican-3 is a useful diagnostic marker for a component of hepatocellular carcinoma in human liver cancer. Int J Oncol. 2009;34(3):649-656.

32. Wang HL, Anatelli F, Zhai QJ, Adley B, Chuang ST, Yang XJ. Glypican-3 as a useful diagnostic marker that distinguishes hepatocellular carcinoma from benign hepatocellular mass lesions. Arch Pathol Lab Med. 2008;132(11):1723-1728.

33. di Tommaso L, Franchi G, Park YN, et al. Diagnostic value of HSP70, glypican 3, and glutamine synthetase in hepatocellular nodules in cirrhosis. Hepatology. 2007;45(3):725-734.

34. Teulon JM, Delcuze Y, Odorico M, et al. Single and multiple bonds in (strept)avidin-biotin interactions. J Mol Recognit. 2011;24(3):490-502.
35. Weber PC, Ohlendorf DH, Wendoloski JJ, Salemme FR. Structural origins of high-affinity biotin binding to streptavidin. Science. 1989; 243(4887):85-88.

36. Holmberg A, Blomstergren A, Nord O, Lukacs M, Lundeberg J, Uhlén $\mathrm{M}$. The biotin-streptavidin interaction can be reversibly broken using water at elevated temperatures. Electrophoresis. 2005;26(3): 501-510.

37. Zhang Y, Chang R, Li M, Zhao K, Zheng H, Zhou X. Docetaxel-loaded lipid microbubbles combined with ultrasound-triggered microbubble destruction for targeted tumor therapy in MHCC-H cells. Onco Targets Ther. 2016;9(Issue 1):4763.

38. Kang J, Wu X, Wang Z, et al. Antitumor effect of docetaxel-loaded lipid microbubbles combined with ultrasound-targeted microbubble activation on VX2 rabbit liver tumors. J Ultrasound Med. 2010;29(1):61-70.

39. Stieger SM, Dayton PA, Borden MA, et al. Imaging of angiogenesis using Cadence contrast pulse sequencing and targeted contrast agents. Contrast Media Mol Imaging. 2008;3(1):9-18.

40. Hummers WS, Offeman RE. Preparation of Graphitic Oxide. J Am Chem Soc. 1958;80(6):1339.

41. Yang K, Zhang S, Zhang G, Sun X, Lee ST, Liu Z. Graphene in mice: ultrahigh in vivo tumor uptake and efficient photothermal therapy. Nano Lett. 2010;10(9):3318-3323.

42. Zhang J, Hu P, Zhang R, et al. Soft-lithographic processed soluble micropatterns of reduced graphene oxide for wafer-scale thin film transistors and gas sensors. J Mater Chem. 2012;22(2):714-718.

43. Tinkov S, Winter G, Coester C, Bekeredjian R. New doxorubicinloaded phospholipid microbubbles for targeted tumor therapy: Part I - Formulation development and in-vitro characterization. J Control Release. 2010;143(1):143-150.

44. Kim J, Kim F, Huang J. Seeing graphene-based sheets. Mater Today. 2010;13(3):28-38.

45. Acik M, Lee G, Mattevi C, Chhowalla M, Cho K, Chabal YJ. Unusual infrared-absorption mechanism in thermally reduced graphene oxide. Nat Mater. 2010;9(10):840-845.

46. Zhang J, Li J, Wang Z, et al. Low-Temperature Growth of Large-Area Heteroatom-Doped Graphene Film. Chemistry of Materials. 2014; 26(7):2460-2466.

47. Wojtoniszak M, Chen X, Kalenczuk RJ, et al. Synthesis, dispersion, and cytocompatibility of graphene oxide and reduced graphene oxide. Colloids Surf B Biointerfaces. 2012;89(1):79-85.

48. Kuo WS, Chang YT, Cho KC, et al. Gold nanomaterials conjugated with indocyanine green for dual-modality photodynamic and photothermal therapy. Biomaterials. 2012;33(11):3270-3278.

49. Rc L, Zhong CN, Rm L. A Multifunctional Anticancer Platform for Multimodal Imaging and Visible Light Driven Photodynamic/Photothermal Therapy. Chem Mater. 2015;(27):1751-1763.

50. Papi M, Palmieri V, Bugli F, et al. Biomimetic antimicrobial cloak by graphene-oxide agar hydrogel. Sci Rep. 2016;6(1):12. 


\section{Supplementary materials}

A
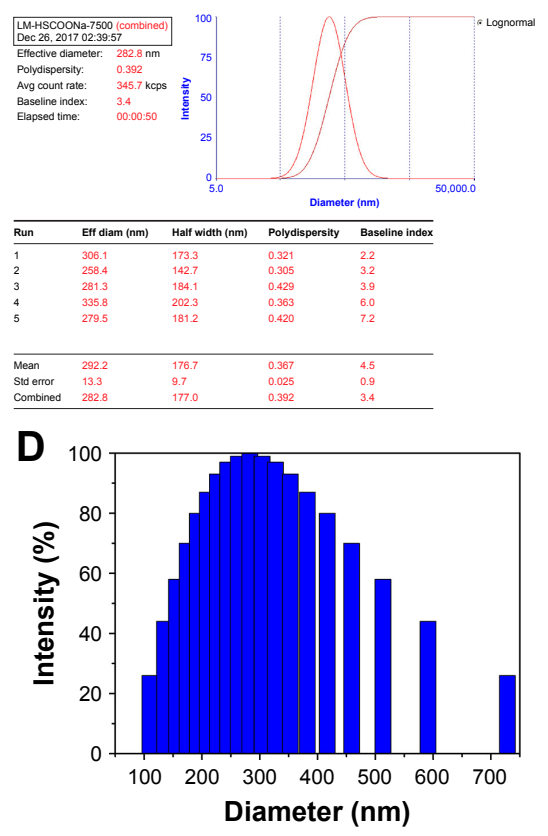

NBs
B

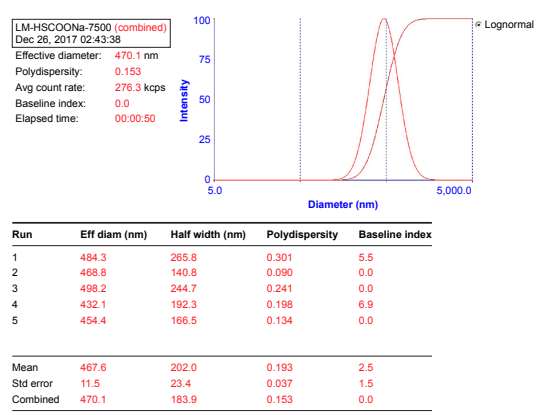

E

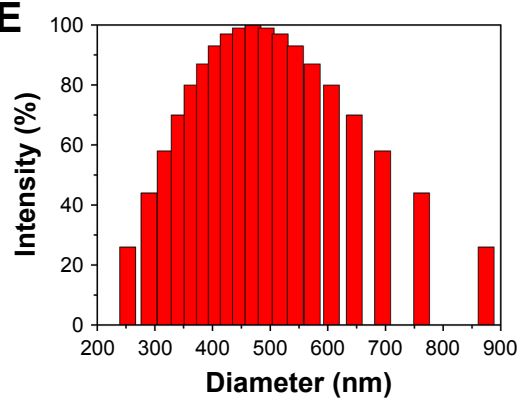

$\square$ Avidinylated NBs
C

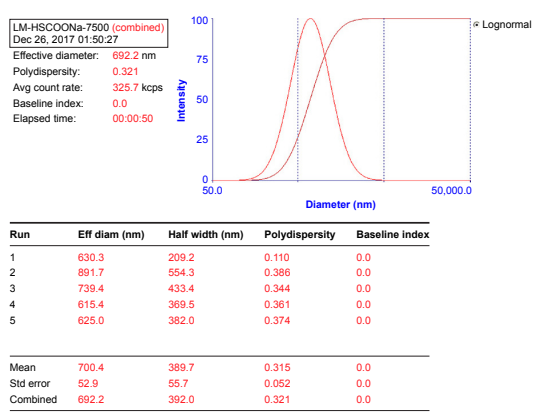

$\mathbf{F}$

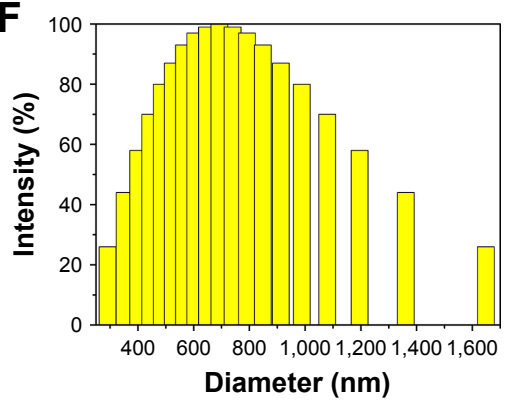

Figure SI Average particle size of NBs (A and D), Avidinylated NBs (B and E) and NBs-GPC3-rGO (C and F) determined by dynamic light scattering (DLS) analysis. The three curves (D-F) of average particle size were analyzed by Shapiro-Wilk test to explain that all three groups of data followed a Gaussian distribution. Abbreviations: GPC3, heparin sulfate proteoglycan glypican-3; NBs, nanobubbles; rGO, reduced graphene oxide.

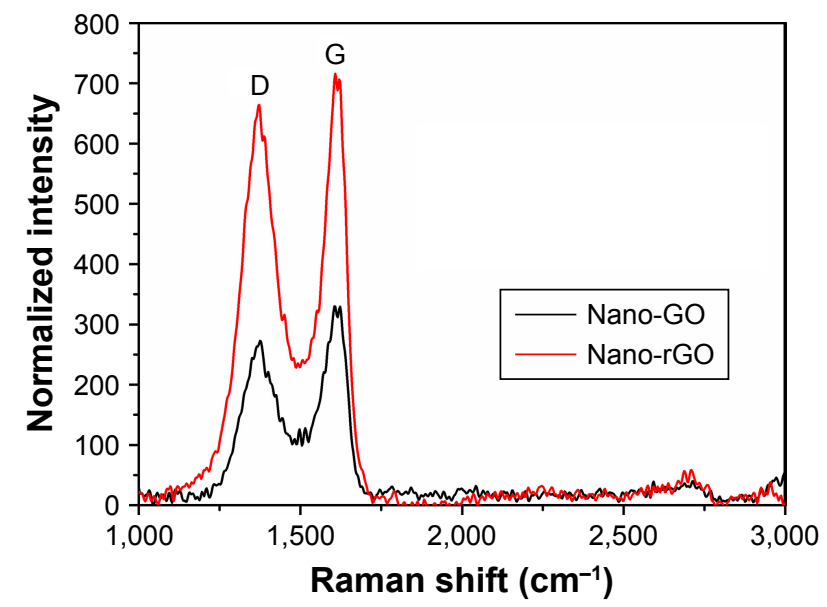

Figure S2 Raman spectroscopy was performed at room temperature using a Raman Microprobe (LabRAM HR800; Horiba Scientific, Jobin-Yvon, France). Note: The $I_{D} / I_{G}$ ratio of nano-rGO did increase notably, indicating that the reduction process altered the structure of G-O with a high quantity of structural defects. Abbreviations: GO, graphene oxide; rGO, reduced graphene oxide. 


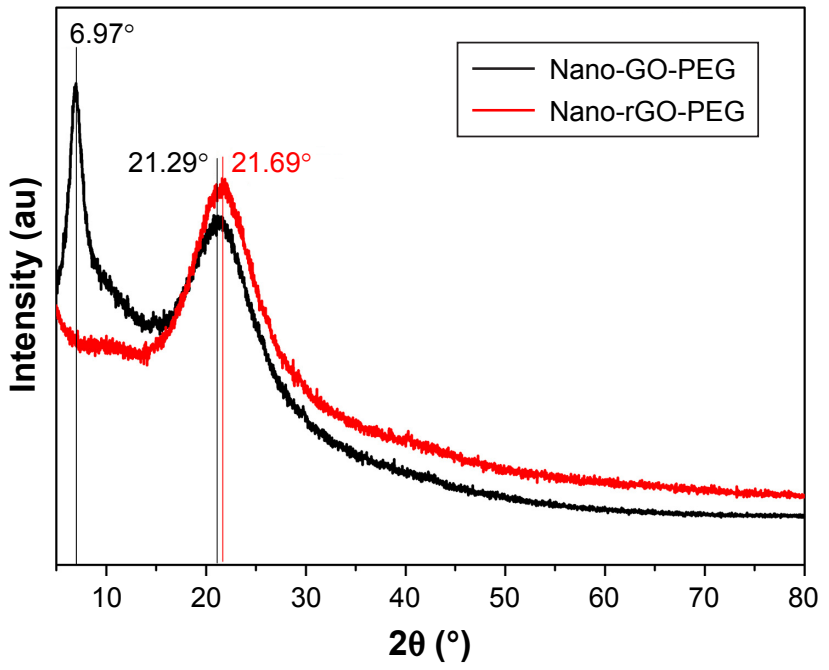

Figure S3 XRD patterns of nano-GO-PEG and nano-rGO-PEG.

Abbreviations: $\mathrm{GO}$, graphene oxide; $r-\mathrm{GO}$, reduced graphene oxide; $\mathrm{XRD}, \mathrm{X}$-ray diffraction.

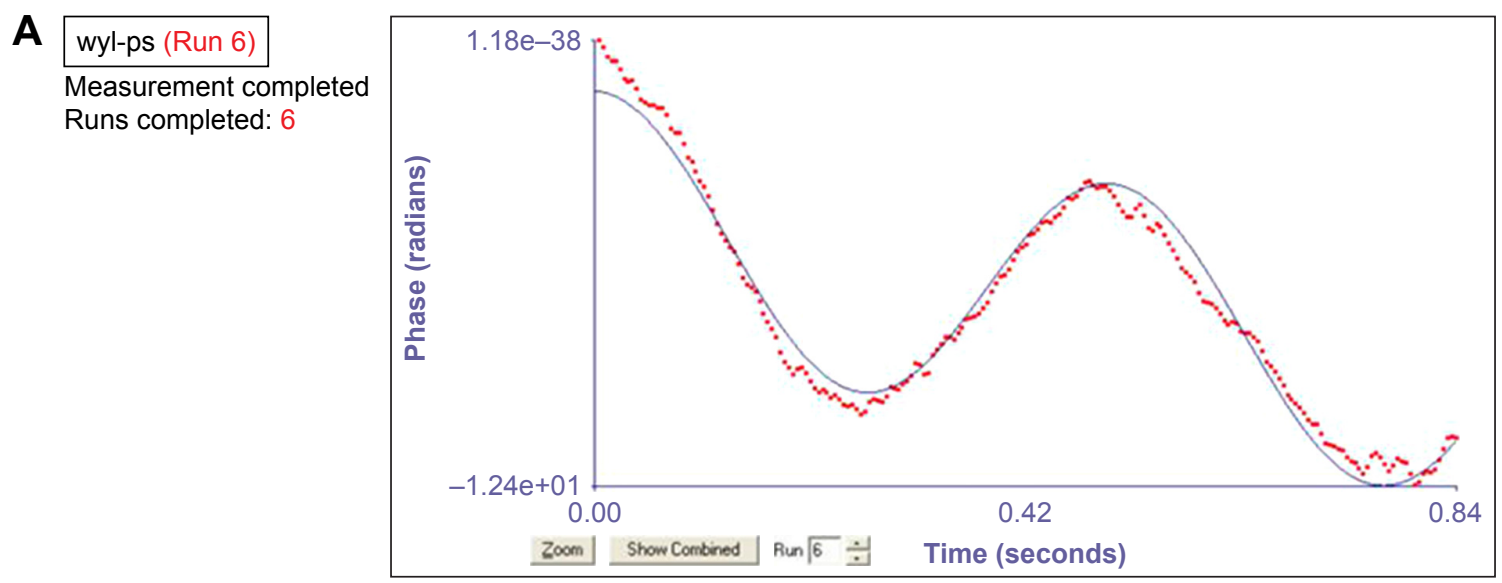

\begin{tabular}{|c|c|c|c|}
\hline Run & Mobility & Zeta potential (mV) & Rel residual \\
\hline 1 & -1.17 & -14.94 & 0.0380 \\
\hline 2 & -2.02 & -25.90 & 0.0726 \\
\hline 3 & -1.75 & -22.41 & 0.0373 \\
\hline 4 & -2.59 & -33.10 & 0.0299 \\
\hline 5 & -2.00 & -25.55 & 0.0663 \\
\hline 6 & -1.91 & -24.46 & 0.0338 \\
\hline Mean & -1.91 & -24.39 & 0.0463 \\
\hline Std error & 0.19 & 2.40 & 0.0075 \\
\hline \multirow[t]{3}{*}{ Combined } & -1.90 & -24.32 & 0.0155 \\
\hline & Stot & Runs & Hide Groph \\
\hline & Cless & Prometers & Copy to clobousd \\
\hline
\end{tabular}

Figure S4 (Continued) 


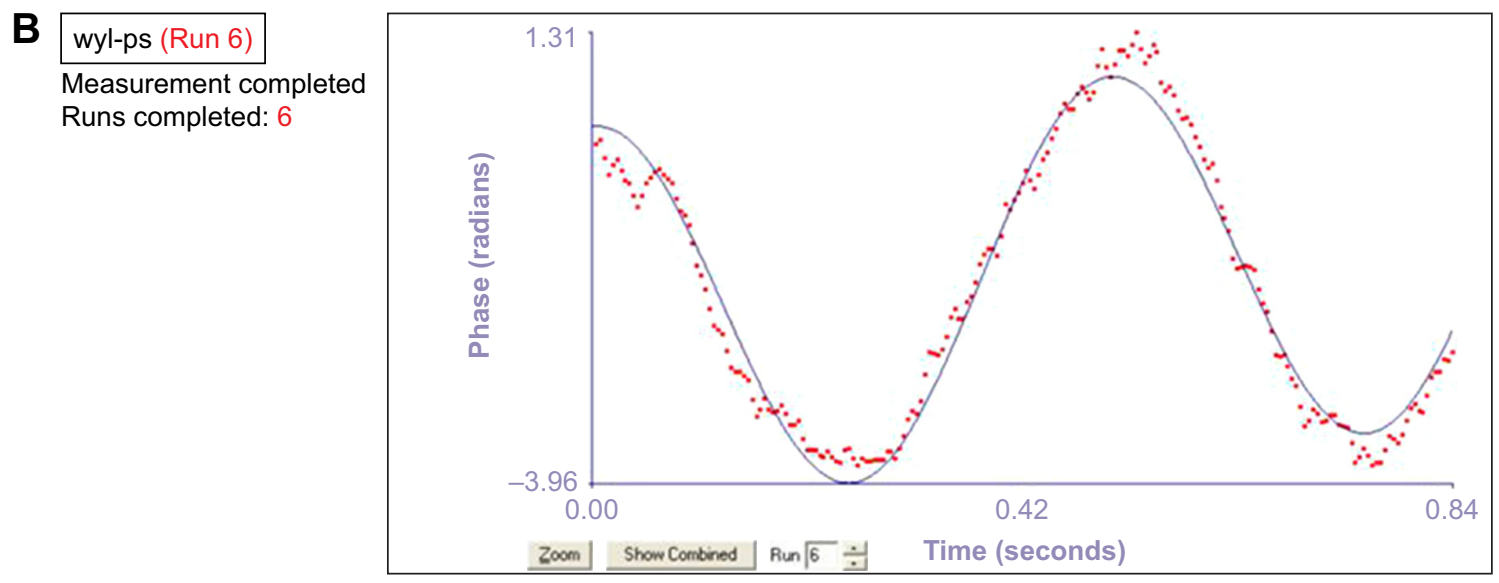

\begin{tabular}{|llll|}
\hline Run & Mobility & Zeta potential $(\mathbf{m V})$ & Rel residual \\
\hline $\mathbf{1}$ & -2.03 & -25.95 & 0.0388 \\
2 & -1.73 & -22.19 & 0.0305 \\
3 & -1.19 & -15.19 & 0.1165 \\
4 & -1.58 & -20.23 & 0.0290 \\
5 & -0.98 & -12.58 & 0.0308 \\
6 & -1.27 & -16.31 & 0.0185 \\
& & & 0.0440 \\
Mean & -1.46 & -18.74 & 0.0147 \\
Std error & 0.16 & 2.02 & 0.0284 \\
Combined & -1.46 & -18.68 & Hise Grosh \\
\hline
\end{tabular}

Figure S4 The zeta potential of the NBs (A) and NBs-GPC3-rGO (B) solution were determined by dynamic light scattering (DLS) measurements. Abbreviations: GPC3, heparin sulfate proteoglycan glypican-3; NBs, nanobubbles; $\mathrm{FGO}$, reduced graphene graphite.

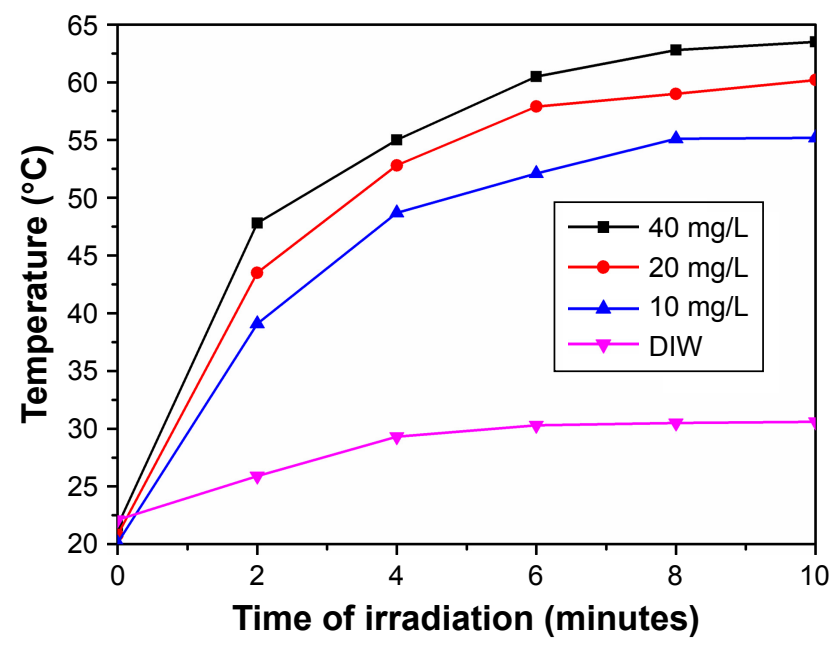

Figure S5 Photothermal heating curves of nano-rGO solutions.

Note: The rise in temperature plots of various concentration of nano-rGO and deionized water (DIW), nano-GO (20 mg/mL) and nano-rGO (20 mg/mL) at $808 \mathrm{~nm}$ at $2 \mathrm{~W} / \mathrm{cm}^{2} \mathrm{NIR}$.

Abbreviations: rGO, reduced graphene graphite; NIR, near infrared. 


\section{Publish your work in this journal}

The International Journal of Nanomedicine is an international, peerreviewed journal focusing on the application of nanotechnology in diagnostics, therapeutics, and drug delivery systems throughout the biomedical field. This journal is indexed on PubMed Central,

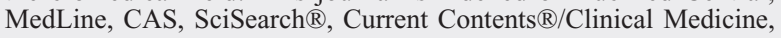

Journal Citation Reports/Science Edition, EMBase, Scopus and the Elsevier Bibliographic databases. The manuscript management system is completely online and includes a very quick and fair peer-review system, which is all easy to use. Visit http://www.dovepress.com/ testimonials.php to read real quotes from published authors.

Submit your manuscript here: http://www.dovepress.com/international-journal-of-nanomedicine-journal 